\title{
On Inverse Boundary-Condition Problems in Radiative Transfer
}

\author{
L. B. Barichello, ${ }^{\dagger}$ R. D. M. Garcia ${ }^{\dagger}$ and C. E. Siewert ${ }^{\S}$ \\ †Instituto de Matemática \\ Universidade Federal do Rio Grande do Sul \\ 91509-900 Porto Alegre, RS \\ Brazil \\ $\ddagger$ Centro Técnico Aeroespacial \\ Instituto de Estudos Avançados \\ 12231-970 São José dos Campos, SP \\ Brazil

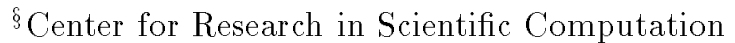 \\ Mathematics Department \\ North Carolina State University \\ Raleigh, NC 27695-8205
}

USA

Abstract

Some elementary computations are reported to suggest that a certain type of inverse boundary-condition problem in radiative transfer can, in some cases, be solved quite simply.

March 5, 1996 


\section{Introduction}

In a recent paper $^{1}$ concerning inverse-source problems in radiative transfer, it was mentioned that inverse boundary-condition problems could be solved in a manner similar to the one used to solve the source problems. Here we report some computations to support that suggestion.

We consider the equation of transfer ${ }^{2}$ for the radiation intensity $I(\tau, \mu)$, written as

$$
\mu \frac{\partial}{\partial \tau} I(\tau, \mu)+I(\tau, \mu)=\frac{\varpi}{2} \sum_{l=0}^{L} \beta_{l} P_{l}(\mu) \int_{-1}^{1} P_{l}\left(\mu^{\prime}\right) I\left(\tau, \mu^{\prime}\right) d \mu^{\prime}
$$

where $\tau \in\left(0, \tau_{0}\right)$ is the optical variable, $\mu \in[-1,1]$ is the cosine of the polar angle (as measured from the positive $\tau$ axis) used to describe the direction of propagation of the radiation and $\varpi$ is the albedo for single scattering. In addition, the $\beta_{l}$ are the coefficients in a Legendre polynomial expansion of the scattering law. For direct problems in radiative transfer, we normally supplement Eq. (1) with boundary conditions of the form

$$
I(0, \mu)=F_{1}(\mu)+\rho_{1}^{s} I(0,-\mu)+2 \rho_{1}^{d} \int_{0}^{1} I\left(0,-\mu^{\prime}\right) \mu^{\prime} d \mu^{\prime}
$$

and

$$
I\left(\tau_{0},-\mu\right)=F_{2}(\mu)+\rho_{2}^{s} I\left(\tau_{0}, \mu\right)+2 \rho_{2}^{d} \int_{0}^{1} I\left(\tau_{0}, \mu^{\prime}\right) \mu^{\prime} d \mu^{\prime}
$$

for $\mu \in(0,1]$. Here $F_{1}(\mu)$ and $F_{2}(\mu)$ are considered given, and $\rho_{\beta}^{s}$ and $\rho_{\beta}^{d}$, for $\beta=1$ and 2 , are coefficients for specular and diffuse reflection.

For the inverse problem considered here, we suppose that the radiation density

$$
\Phi(\tau)=\int_{-1}^{1} I(\tau, \mu) d \mu
$$

is known at certain positions $\left\{\tau_{i}\right\}$ within the medium, and we then seek to determine what functions $F_{1}(\mu)$ and $F_{2}(\mu)$ for $\mu \in(0,1]$ can induce such a radiation density. ${ }^{3}$ The motivation for this problem comes from the field of radiation therapy, where it is often a difficult task to predict the angular shape and strength of an exposing radiation beam incident on the surface of the body that will give rise to a desired internal dose. We note that the analysis of practical applications of radiation therapy must, in general, be based on a more complex model than the one considered here. Of course, more complex mathematical models may require strictly numerical methods of solution or Monte Carlo methods, coupled with iterative procedures, and thus, the simplified model considered here is to be understood as a first attempt to provide a more deterministic algorithm for the problem. Also, in order to allow for some possible radiative heat-transfer applications, we use in Eqs. (2) the coefficients $\rho_{\beta}^{s}$ and $\rho_{\beta}^{d}$, for $\beta=1$ and 2 , to include the effects of specular and diffuse reflection at the boundaries.

March 5, 1996 


\section{Formulation}

To solve the considered inverse problem, we first formulate and solve the two sets of direct problems expressed, for $k=0,1,2, \ldots, K_{\alpha}, \alpha=1$ and 2 , by the equation of transfer

$$
\mu \frac{\partial}{\partial \tau} \Upsilon_{k}^{\alpha}(\tau, \mu)+\Upsilon_{k}^{\alpha}(\tau, \mu)=\frac{\varpi}{2} \sum_{l=0}^{L} \beta_{l} P_{l}(\mu) \int_{-1}^{1} P_{l}\left(\mu^{\prime}\right) \Upsilon_{k}^{\alpha}\left(\tau, \mu^{\prime}\right) d \mu^{\prime}
$$

for $\tau \in\left(0, \tau_{0}\right)$ and $\mu \in[-1,1]$, and the boundary conditions

$$
\Upsilon_{k}^{\alpha}(0, \mu)=\delta_{1, \alpha} H_{k}(\mu)+\rho_{1}^{s} \Upsilon_{k}^{\alpha}(0,-\mu)+2 \rho_{1}^{d} \int_{0}^{1} \Upsilon_{k}^{\alpha}\left(0,-\mu^{\prime}\right) \mu^{\prime} d \mu^{\prime}
$$

and

$$
\Upsilon_{k}^{\alpha}\left(\tau_{0},-\mu\right)=\delta_{2, \alpha} H_{k}(\mu)+\rho_{2}^{s} \Upsilon_{k}^{\alpha}\left(\tau_{0}, \mu\right)+2 \rho_{2}^{d} \int_{0}^{1} \Upsilon_{k}^{\alpha}\left(\tau_{0}, \mu^{\prime}\right) \mu^{\prime} d \mu^{\prime}
$$

for $\mu \in(0,1]$. Here we use $\left\{H_{k}(\mu)\right\}$ to denote a set of basis functions that we are free to specify. If we now solve these two sets of basic problems and determine, for $k=0,1,2, \ldots, K_{\alpha}, \alpha=1$ and 2 ,

$$
\Xi_{k}^{\alpha}(\tau)=\int_{-1}^{1} \Upsilon_{k}^{\alpha}(\tau, \mu) d \mu
$$

we can approximate the radiation density defined in Eq. (3) by

$$
\widehat{\Phi}(\tau)=\sum_{k=0}^{K_{1}} a_{k} \Xi_{k}^{1}(\tau)+\sum_{k=0}^{K_{2}} b_{k} \Xi_{k}^{2}(\tau),
$$

where the constants $\left\{a_{k}\right\}$ and $\left\{b_{k}\right\}$ are to be found simply by constraining $\widehat{\Phi}(\tau)$ to match the desired radiation density $\Phi(\tau)$ at $K_{1}+K_{2}+2$ positions given by $\left\{\tau_{i}\right\}$. We get

$$
\sum_{k=0}^{K_{1}} a_{k} \Xi_{k}^{1}\left(\tau_{i}\right)+\sum_{k=0}^{K_{2}} b_{k} \Xi_{k}^{2}\left(\tau_{i}\right)=\Phi\left(\tau_{i}\right)
$$

for $i=1,2, \ldots, K_{1}+K_{2}+2$. Once this system of linear algebraic equations is solved for $\left\{a_{k}\right\}$ and $\left\{b_{k}\right\}$, it follows that we can compute the desired boundary data from

$$
F_{1}(\mu)=\sum_{k=0}^{K_{1}} a_{k} H_{k}(\mu)
$$

and

$$
F_{2}(\mu)=\sum_{k=0}^{K_{2}} b_{k} H_{k}(\mu)
$$

for $\mu \in(0,1]$. We note that our solution given by Eqs. (9) is independent of the method to be used to solve the basic problems defined by Eqs. (4) and (5).

March 5, 1996 


\section{A Spherical-Harmonics Solution}

We now develop a spherical-harmonics $\left(P_{N}\right)$ solution for the two sets of direct problems that are fundamental to the solution of our inverse problem.

Following previous works, ${ }^{4,5}$ we begin by writing our $P_{N}$ approximation (with $\mathrm{N}$ odd) to $\Upsilon_{k}^{\alpha}(\tau, \mu)$ as

$$
\Upsilon_{k}^{\alpha}(\tau, \mu)=\sum_{n=0}^{N}\left(\frac{2 n+1}{2}\right) P_{n}(\mu) \sum_{j=1}^{J}\left[A_{k, j}^{\alpha} e^{-\tau / \xi_{j}}+(-1)^{n} B_{k, j}^{\alpha} e^{-\left(\tau_{0}-\tau\right) / \xi_{j}}\right] g_{n}\left(\xi_{j}\right)
$$

where $J=(N+1) / 2, P_{n}(\mu)$ and $g_{n}(\xi)$ are respectively the Legendre and the Chandrasekhar polynomials of

order $n$, the eigenvalue $\xi_{j}$ is the $j$-th positive zero of $g_{N+1}(\xi)$ and $\left\{A_{k, j}^{\alpha}\right\}$ and $\left\{B_{k, j}^{\alpha}\right\}$ are unknown coefficients. If we now substitute Eq. (10) into the Mark version ${ }^{6}$ of the boundary conditions expressed by Eqs. (5), we obtain the linear systems, for $k=0,1,2, \ldots, K_{\alpha}, \alpha=1$ and 2 ,

$$
\left(\begin{array}{cc}
\mathbf{M}^{1} & \mathbf{N}^{1} \mathbf{E} \\
\mathbf{N}^{2} \mathbf{E} & \mathbf{M}^{2}
\end{array}\right)\left(\begin{array}{c}
\mathbf{A}_{k}^{\alpha} \\
\mathbf{B}_{k}^{\alpha}
\end{array}\right)=\left(\begin{array}{c}
\delta_{1, \alpha} \mathbf{H}_{k} \\
\delta_{2, \alpha} \mathbf{H}_{k}
\end{array}\right)
$$

Here, the vectors that define the vector of unknowns are given by

$$
\mathbf{A}_{k}^{\alpha}=\left(\begin{array}{c}
A_{k, 1}^{\alpha} \\
A_{k, 2}^{\alpha} \\
\vdots \\
A_{k, J}^{\alpha}
\end{array}\right) \quad \text { and } \quad \mathbf{B}_{k}^{\alpha}=\left(\begin{array}{c}
B_{k, 1}^{\alpha} \\
B_{k, 2}^{\alpha} \\
\vdots \\
B_{k, J}^{\alpha}
\end{array}\right)
$$

and the vector that defines the right-hand side is given by

$$
\mathbf{H}_{k}=\left(\begin{array}{c}
H_{k}\left(\mu_{1}\right) \\
H_{k}\left(\mu_{2}\right) \\
\vdots \\
H_{k}\left(\mu_{J}\right)
\end{array}\right)
$$

where $\mu_{i}, i=1,2, \ldots, J$, denote the positive zeros of $P_{N+1}(\mu)$. In addition, $\mathbf{E}$ is a diagonal matrix of order $J$ with $\exp \left(-\tau_{0} / \xi_{j}\right)$ as the $j$-th diagonal element, and by defining

$$
\begin{gathered}
S_{i, j}=\sum_{n=0}^{N}\left(\frac{2 n+1}{2}\right) P_{n}\left(\mu_{i}\right) g_{n}\left(\xi_{j}\right), \\
T_{i, j}=\sum_{n=0}^{N}(-1)^{n}\left(\frac{2 n+1}{2}\right) P_{n}\left(\mu_{i}\right) g_{n}\left(\xi_{j}\right), \\
U_{j}=\sum_{n=0}^{N}\left(\frac{2 n+1}{2}\right) \Delta_{n} g_{n}\left(\xi_{j}\right)
\end{gathered}
$$

and

$$
V_{j}=\sum_{n=0}^{N}(-1)^{n}\left(\frac{2 n+1}{2}\right) \Delta_{n} g_{n}\left(\xi_{j}\right)
$$

March 5, 1996 
with

$$
\Delta_{n}=\int_{0}^{1} P_{n}(\mu) \mu d \mu
$$

we can write the elements of the $J \times J$ matrices $\mathbf{M}^{\beta}$ and $\mathbf{N}^{\beta}, \beta=1$ or 2 , as

$$
M_{i, j}^{\beta}=S_{i, j}-\rho_{\beta}^{s} T_{i, j}-2 \rho_{\beta}^{d} V_{j}
$$

and

$$
N_{i, j}^{\beta}=T_{i, j}-\rho_{\beta}^{s} S_{i, j}-2 \rho_{\beta}^{d} U_{j}
$$

In regard to the $\Delta_{n}$ constants defined by Eq. (16) and required in Eqs. (15), we note that a particularly simple and accurate scheme for computing these constants is provided by $\Delta_{0}=1 / 2, \Delta_{1}=1 / 3$ and, for $m=1,2, \ldots, J-1$,

$$
\Delta_{2 m}=-\left(\frac{2 m-3}{2 m+2}\right) \Delta_{2 m-2}
$$

and

$$
\Delta_{2 m+1}=0 .
$$

Now, since the matrix of coefficients in Eq. (11) is the same for any $k=0,1,2, \ldots, K_{\alpha}, \alpha=1$ and 2 , it is clear that only one LU factorization is sufficient for solving all the $K_{1}+K_{2}+2$ systems defined by Eq. (11). In this work, we have used either DGECO or DGEFA of the LINPACK subroutine package ${ }^{7}$ to factor the matrix of coefficients, followed by DGESL to solve the linear systems. Having determined the coefficients $\left\{A_{k, j}^{\alpha}\right\}$ and $\left\{B_{k, j}^{\alpha}\right\}$, we can integrate Eq. (10) to find that our $P_{N}$ approximation to $\Xi_{k}^{\alpha}(\tau)$, required in Eq. (8) for $k=0,1,2, \ldots, K_{\alpha}, \alpha=1$ and 2 , and $\tau=\tau_{i}, i=1,2, \ldots, K_{1}+K_{2}+2$, is given by

$$
\Xi_{k}^{\alpha}(\tau)=\sum_{j=1}^{J}\left[A_{k, j}^{\alpha} e^{-\tau / \xi_{j}}+B_{k, j}^{\alpha} e^{-\left(\tau_{0}-\tau\right) / \xi_{j}}\right] .
$$

\section{Sample Calculations}

To have a specific scattering law for testing our solution technique, and to avoid having to provide a table of the scattering law coefficients $\left\{\beta_{l}\right\}$, we use here the binomial scattering law ${ }^{8}$

$$
p(\cos \Theta)=\frac{L+1}{2^{L}}(1+\cos \Theta)^{L}
$$

which can be represented exactly with $L+1$ Legendre coefficients that can be computed with $\beta_{0}=1$ and ${ }^{9}$

$$
\beta_{l}=\left(\frac{2 l+1}{2 l-1}\right)\left(\frac{L+1-l}{L+1+l}\right) \beta_{l-1} .
$$

March 5, 1996 
For our first sample calculation, we considered a case where there is no radiation inciding on the right boundary, i.e. $F_{2}(\mu)=0$. To deal with this case, we note that we used $K_{2}=-1$ in our formalism, so that the second set of direct problems defined by Eqs. (4) and (5) with $\alpha=2$ was not required and the summations involving $\left\{b_{k}\right\}$ in Eqs. (7) and (8) could be ignored when solving this problem. Then with $L=99, \varpi=0.99$, $\tau_{0}=1.0,10.0$ and 100.0, and various choices for the reflection coefficients $\rho_{\beta}^{s}$ and $\rho_{\beta}^{d}$, we solved, using the spherical-harmonics method with Mark boundary conditions described in Sec. 3, the direct problems defined by Eq. (4) subject to Eqs. (5) with

$$
H_{k}(\mu)=P_{2 k}(\mu)
$$

for $k=0,1, \ldots, K_{1}$ and various values of $K_{1}$. Note that we continue to use $P_{n}(x)$ to denote the Legendre polynomial of order $n$. Next, we used

$$
\left.\tau_{i}=\left[(i-1) / K_{1}\right)\right] \tau_{*}, \quad i=1,2, \ldots, K_{1}+1,
$$

where $\tau_{*}=\min \left\{10.0, \tau_{0}\right\}$, and considered a radiation density specified by

$$
\Phi\left(\tau_{i}\right)=\sum_{k=0}^{K_{1}} \Xi_{k}^{1}\left(\tau_{i}\right)
$$

on the right-hand side of Eq. (8) to define a system of linear algebraic equations for the desired $\left\{a_{k}\right\}$. It is clear that the choice of the observation points $\left\{\tau_{i}\right\}$ can affect greatly the condition number of the linear system given by Eq. (8). And, though we have not made a definitive study of this issue, we did find that the scheme defined by Eq. (23) provided an improvement, for thick layers, over using a uniform distribution. We note that, for $K_{1} \leq 5$, we were able to obtain the correct results, $a_{k}=1.0$ for $k=0,1, \ldots, K_{1}$. As we increased $K_{1}$ beyond 5 , we observed a progressive deterioration in the conditioning of the linear system for $\left\{a_{k}\right\}$.

Of course, there are applications for which we would like $\Phi(\tau)$ to display a certain shape, the simplest being that of a constant. However, upon repeating the first calculation with $\Phi\left(\tau_{i}\right)=1.0$ on the right-hand side of Eq. (8), we found that the resulting $F_{1}(\mu)$ was not non-negative (clearly a non-physical result.)

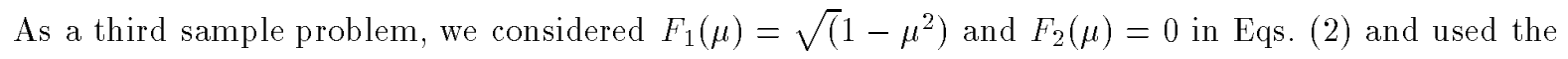
spherical-harmonics method described in Sec. 3 to solve the direct problem defined by Eqs. (1) and (2) with $L=99, \varpi=0.99$ and various choices for the reflection coefficients $\rho_{\beta}^{s}$ and $\rho_{\beta}^{d}$, for $\tau_{0}=1.0,10.0$ and 100.0. The solution to this problem was computed for the $\tau_{i}$ given by Eq. (23), in order to specify the radiation density $\Phi\left(\tau_{i}\right)$ required in Eq. (8). In addition, we solved the direct problems defined by using the same basic 
data $\left(L, \varpi, \rho_{\beta}^{s}, \rho_{\beta}^{d}\right.$ and $\left.\tau_{0}\right)$ in Eqs. (4) and (5) for $\alpha=1$ and the basis functions specified by Eq. (22) in Eqs. (5), and then we used these solutions to compute the quantities $\left\{\Xi_{k}^{1}\left(\tau_{i}\right)\right\}$ required in Eq. (8). Here, as in the first sample problem, we took $K_{2}=-1$ in our formalism, so that the set of direct problems for $\alpha=2$ was not required and the summations involving $\left\{b_{k}\right\}$ in Eqs. (7) and (8) were also ignored. Having solved the system of equations for the desired $\left\{a_{k}\right\}, k=0,1, \ldots, K_{1}$, with $K_{1}=5$, we then used Eq. (7) to recompute the radiation density at the values of $\tau$ given by Eq. (23) and found (not surprisingly) agreement to at least five figures with the originally computed direct density, even though there was, of course, some difference between the assumed $F_{1}(\mu)$ and the one computed.

Finally, in order to give some specific results, we consider the case defined by

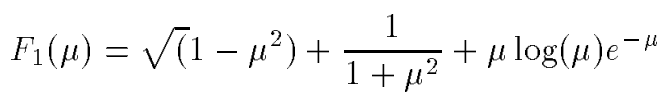

and $F_{2}(\mu)=0$ along with $\varpi=0.99, \tau_{0}=10.0, L=99, \rho_{1}^{s}=0.1, \rho_{1}^{d}=0.2, \rho_{2}^{s}=0.3$, and $\rho_{2}^{d}=0.4$. We used the $P_{199}$ solution to solve the basic problems and then used $K_{1}=9$ to obtain the results shown in Table 1 . Again we see that while the values of $F_{1}(\mu)$ computed from the inverse method are not very accurate, the values of the radiation density resulting from the use of the approximate $F_{1}(\mu)$ match the correct values to within \pm 1 in the sixth significant figure.

\section{Concluding Remarks}

We start this section by reporting some observations in regard to the numerical aspects of our solution. By monitoring the condition number estimates returned by DGECO, we have found, for the sample calculations reported in Sec. 4, that the linear systems defined by Eq. (11) were always well-conditioned, even in very high order, say $N=499$. On the other hand, as discussed in Sec. 4, the linear system defined by Eq. (8) became poorly conditioned for $K_{1}+K_{2}+2$ typically between 10 and 15 . For the third and fourth sample problems, we have observed that while the boundary conditions computed from the inverse method did not agree very accurately with the postulated ones, the values of the computed radiation density matched very closely the correct values. However, it is possible that for more complicated problems the inherent ill-conditioning of Eq. (8) may pose a more severe limitation on the level of accuracy attainable for the computed radiation density.

Finally, we conclude by noting that, as with other types of inverse problems in radiative transfer, the existence and uniqueness issues play also an important role here. As seen for the second sample problem considered in Sec. 4, simply postulating a desired $\Phi(\tau)$ for a given problem may give rise to non-physical 
results for the boundary conditions. The question of assuring that a preassigned radiation density will (or will not) give rise to a physically acceptable pair of boundary conditions remains an open one.

\section{Acknowledgements}

One of the authors (L.B.B.) would like to express her thanks to the Mathematics Department of North Carolina State University for the kind hospitality extended throughout a period during which this work was

done. In addition, it is noted that the work of L.B.B. and R.D.M.G. was supported in part by CNPq of Brazil.

\section{References}

1. C. E. Siewert, JQSRT 50, 603 (1993).

2. S. Chandrasekhar, Radiative Transfer, Oxford University Press, London (1950).

3. L. B. Barichello and M. T. de Vilhena, Proc. IX ENFIR, p. 22, Caxambu, MG, Brazil (1993).

4. M. Benassi, R. D. M. Garcia, A. H. Karp and C. E. Siewert, Astrophys. J. 280, 853 (1984).

5. R. D. M. Garcia, C. E. Siewert and J. R. Thomas, Jr., Trans. Am. Nucl. Soc. 71, 220 (1994).

6. B. Davison, Neutron Transport Theory, Oxford University Press, London (1957).

7. J. J. Dongarra, J. R. Bunch, C. B. Moler and G. W. Stewart, LINPACK Users' Guide, SIAM, Philadelphia (1979).

8. H. G. Kaper, J. K. Shultis and J. G. Veninga, J. Comp. Phys. 6, 288 (1970).

9. N. J. McCormick and R. Sanchez, J. Math. Phys. 22, 199 (1981). 
Table 1. Direct and Inverse Results for Boundary Data and Radiation Density

\begin{tabular}{|ccccc|}
\hline$\xi$ & $F_{1}(\xi=\mu)$ & $\widehat{F}_{1}(\xi=\mu)$ & $\Phi\left(\xi=\tau / \tau_{0}\right)$ & $\widehat{\Phi}\left(\xi=\tau / \tau_{0}\right)$ \\
\hline 0.00 & 2.00 & 1.88 & 2.51815 & 2.51815 \\
0.10 & 1.78 & 1.80 & 2.23102 & 2.23103 \\
0.20 & 1.68 & 1.67 & 2.11349 & 2.11349 \\
0.30 & 1.60 & 1.60 & 2.02838 & 2.02838 \\
0.40 & 1.53 & 1.54 & 1.96096 & 1.96096 \\
0.50 & 1.46 & 1.45 & 1.90537 & 1.90537 \\
0.60 & 1.37 & 1.37 & 1.85848 & 1.85848 \\
0.70 & 1.26 & 1.26 & 1.81829 & 1.81829 \\
0.80 & 1.13 & 1.13 & 1.78315 & 1.78315 \\
0.90 & 0.95 & 0.94 & 1.75087 & 1.75087 \\
1.00 & 0.50 & 0.47 & 1.70421 & 1.70421 \\
\hline
\end{tabular}

